\title{
Effects of diel vertical migration on ephippia production in Daphnia
}

\author{
Victor ALEKSEEV \\ Zoological Institute of the Russian Academy of Sciences, University emb., 1, 199034, St. Petersburg, Russia \\ e-mail address: alekseev@zin.ru
}

\begin{abstract}
Fish presence in experimentally simulated temperature conditions (limno-towers) led to diel vertical migration and resulted in a decrease of ephippia production in Daphnia pulicaria. Diel fluctuation of food, temperature and day length similar to those experienced by migrating Daphnia were tested in laboratory experiments with flow-through-systems. Daphnids were kept under these conditions for 15 days and the proportions of females producing an ephippium were determined. In addition, maturation time, survival to maturation, size of the first clutch and female dry weight (without eggs) on day 15 were traced. The most important factor affecting ephippia production in migrating Daphnia was photoperiod change, and when mother and embryo are exposed to alternating light conditions, these are thought to have the effect on the mother of acting as a signal to stop ephippia production. Such effects might be explained by the different sensitiveness to light intensity in females carrying an embryo and an embryo itself in broods. Fish presence forced Daphnia to stay in low-light conditions during daytime hours, to avoid attacks by fish. The Daphnia were able to check light intensity constantly by short vertical jumps above a light-threshold that was confirmed experimentally in limno-towers. The dim conditions were possibly light enough for adults to check day length, but were too dark for embryos shaded by the mother's body. Food conditions played a relatively small role in the process, and no effects of temperature on ephippia production were found. As expected, food affected the size of the first clutch, and temperature controlled the time to maturation. Photoperiod had a marginally significant influence on the time to maturation in Daphnia. A hypothesis on the role of photoperiod as the key factor for Daphnia life cyclic recurrence and other seasonal adaptations is proposed.
\end{abstract}

Key words: diapause, photoperiod, maternal effect, fish kairomones, diel vertical migration, ephippia production

\section{INTRODUCTION}

Diapause in zooplankton is supposed to be a strategy for surviving adverse conditions (Hutchinson 1967). Hatching from the egg bank is a mechanism to ensure recruitment after periods of harsh conditions, e.g., winter, insufficient food, and intense fish predation, as well as to create a long-term generation overlap which maintains genetic diversity (Hairston 1996). The production of ephippia in Daphnia is a particularly wellstudied example of such a survival strategy. Since ephippia formation needs some time, female Daphnia must anticipate changing environmental conditions in order to produce resting eggs at the appropriate time (Taylor 1980).

Seasonal events in deep lakes are fairly predictable (Sommer et al. 1986), hence factors correlated to season (e.g., photoperiod) are reliable cues for the induction of ephippia (Stross \& Hill 1965). Unpredictable events require an immediate reaction to cues signalling environmental change. Chemical cues have been found to trigger such responses (Tollrian \& Harvell 1999). With respect to ephippia production, a chemical factor produced by crowding daphniids may signal future deterioration of the food conditions (Kleiven et al. 1992), and a kairomone released by fish can indicate fish predation (Pijanowska \& Stolpe 1996; Slusarczyk 1995). These authors consider resting egg production a defence strategy against predation, as ephippia pass through fish guts unharmed (Mellors 1975). Although fish predation is an important force structuring lake zooplankton communities, the induction of sexual reproduction in Daphnia by a fish kairomone has so far been demonstrated for a single clone of $D$. magna from a very shallow lake (Pijanowska \& Stolpe 1996; Slusarczyk 1995). In contrast, Spaak \& Boersma (2001) found that the presence of fish kairomone in deep mesocosms suppressed the production of ephippia when lake daphniids performed diel vertical migrations (DVM). They discussed the idea that DVM in deep lakes may be a less costly defence strategy against fish predation than ephippia production, if a refuge from predation is available.

Ephippia production in deep lakes seems to be concentrated in two periods, late spring and autumn (Caceres 1998; Spaak 1995), hence it ceases during the summer period when daphniids perform DVM. However, this observation is not sufficient to construct a causal relationship.

The proximate factors controlling DVM and ephippia production have been well studied. The seasonal pattern of DVM can be explained by fish activity (Lampert 1993), but the reasons for the seasonal pattern of ephippia production are not yet completely clear. Alekseev \& Lampert (2001) explained the bimodal seasonal pattern by maternal effects of food and photoperiod. An inverse correlation between DVM and ephippia production, however, would lead to the same pattern. I started this study to unravel the mechanisms which may 
link DVM and the cessation of ephippia production in summer.

A direct effect of fish kairomone causing opposite reactions in shallow and deep lake Daphnia cannot be ruled out, but is unlikely. There may rather be an indirect effect of DVM on the cues triggering ephippia production. Two possible mechanisms may be involved, considering that migrating Daphnia spend most of the day under low-light conditions: (1) migrating Daphnia may perceive a change in photoperiod, thus the change in ephippia production is caused by the known photoperiod response, or (2) the tendency to produce ephippia depends on the light intensity, i.e., ephippia production requires high light levels. The latter hypothesis is favoured by Slusarczyk et al. (2004, this issue) for shallow lake D. magna that lack a deep-water refuge. We used the same clone of D. pulicaria as Alekseev \& Lampert (2001) and combined experiments in the deep mesocosms used by Spaak \& Boersma (2001) with laboratory experiments to study the problem in detail.

\section{METHODS}

\subsection{Mesocosm experiments}

Large-scale experiments were carried out in the Plön Plankton Tower system, two large stainless steel mesocosms with controlled vertical stratification (Lampert \& Loose 1992). The thermocline was set to $7.5 \mathrm{~m}$ depth. It divided the water column into an upper layer of $20^{\circ} \mathrm{C}$ and a lower layer of $15^{\circ} \mathrm{C}$, with the transition zone between 7 and $8 \mathrm{~m}$. Both towers were inoculated with Daphnia pulicaria. Scenedesmus obliquus cultured in large batches in dilute (1:4) Z4 medium (Zehnder \& Gorham 1960) was added as food. The particle concentration in the towers was monitored daily by a CASY (Schärfe, Germany) particle counter, and replenished with fresh algae to maintain a concentration of 0.2-0.3 $\mathrm{mg} \mathrm{C} \mathrm{l}^{-1}$, which is below the incipient limiting concentration for Daphnia (Lampert 1987).

The main illumination came from large lamps with a near-sunlight spectrum that produced at the water surface about $45 \mu$ moles quanta $\mathrm{m}^{-2} \mathrm{~s}^{-1}$ or about of one third of maximal sunlight for this latitude. The photoperiod was 16 hours light and 8 hours dark. Dawn and dusk were simulated by three smaller lamps above each tower that were switched on successively each with 15 minutes delay before the main light started, and were switched off in the reverse order after the main light was turned off. Light within the towers was checked with a LiCor quantum radiometer and by Secchi disk transparency. Although transparency varied with algal density, twice the Secchi disk depth was almost in any case located above the thermocline, which corresponded to night conditions (0.01-0.02 $\mu$ moles quanta $\mathrm{m}^{-2} \mathrm{~s}^{-1}$ ).

The experiment began with the inoculation of both towers with about 12,000 D. pulicaria on 30 April 2002. Three weeks later they had established large populations and began controlling the phytoplankton. At this point, fish kairomones were added to tower 1 by circulating water from an external aquarium holding 20 juvenile (6$8 \mathrm{~g}$ fresh weight) perch (Perca fluviatilis). As this did not result in a clear diel vertical migration, seven freeranging fishes were introduced into the tower on day 10 , which stimulated vertical migration. To avoid excessive feeding on Daphnia, the fish were then transferred into a wire cage deployed at $3.5 \mathrm{~m}$ depth. Only a single fish escaped and remained in the tower free-ranging for the whole experiment.

Tower 2 remained fish-free until day 24, when the fish cage was transferred from tower 1 to tower 2 . The cage was again moved to tower 1 for 4 days from day 32-35 and back to tower 2 for the rest of this experimental series. The series was terminated after 43 days; the towers were drained and tower 2 was refilled and again inoculated. In the second series, the fish cage was deployed in tower 2 on day 12 . On day 26 it was moved close to the surface, where the fish had low impact on the daphniids, and then returned to $3.5 \mathrm{~m}$ on day 32 .

Daphnia were sampled by vertical tows of a plankton net during day and night every five days, and more frequently after the fish conditions were changed. Preserved samples were counted under a dissecting microscope and the proportion of females carrying ephippia determined. To test the reaction of Daphnia to the presence of fish, their vertical distribution was checked on several occasions at noon and midnight. Daphniids were sampled by screening 48 litres of water at each of five depths $(1.5,3.5,5.0,7.0$ and $9.0 \mathrm{~m})$ simultaneously through glass traps (Lampert \& Loose 1992).

\subsection{Reproductive status in the towers}

Changes in abundance of ephippial females in the presence of fish in the towers might be caused by a different status of the females or by selective predation on ephippia. I therefore tested the reproductive status of young females with respect to ephippia induction in successive phases of the tower experiments. Daphniids were collected by vertical tows from the towers at 5-day intervals. Young females of a size close to maturation, but still without eggs, were selected by pipette and placed in $100-\mathrm{ml}$ jars in batches of 10 . Ten replicates (i.e., a sum total of 100 females) were set up. The females were then treated with a complex of diapause-inducing factors (Alekseev \& Lampert 2001) and the proportion of females producing ephippia in the successive 5 days was recorded. The percentage of ephippial females in the jars was then compared to the percentage of ephippial females in the towers on the sampling day following the collection.

\subsection{Factors influencing ephippia production}

I performed a factorial experiment to test the effect of changing environmental factors on the production of ephippia, as they may be experienced by migrating Daphnia. Daphniids were cultivated in the flow-through 

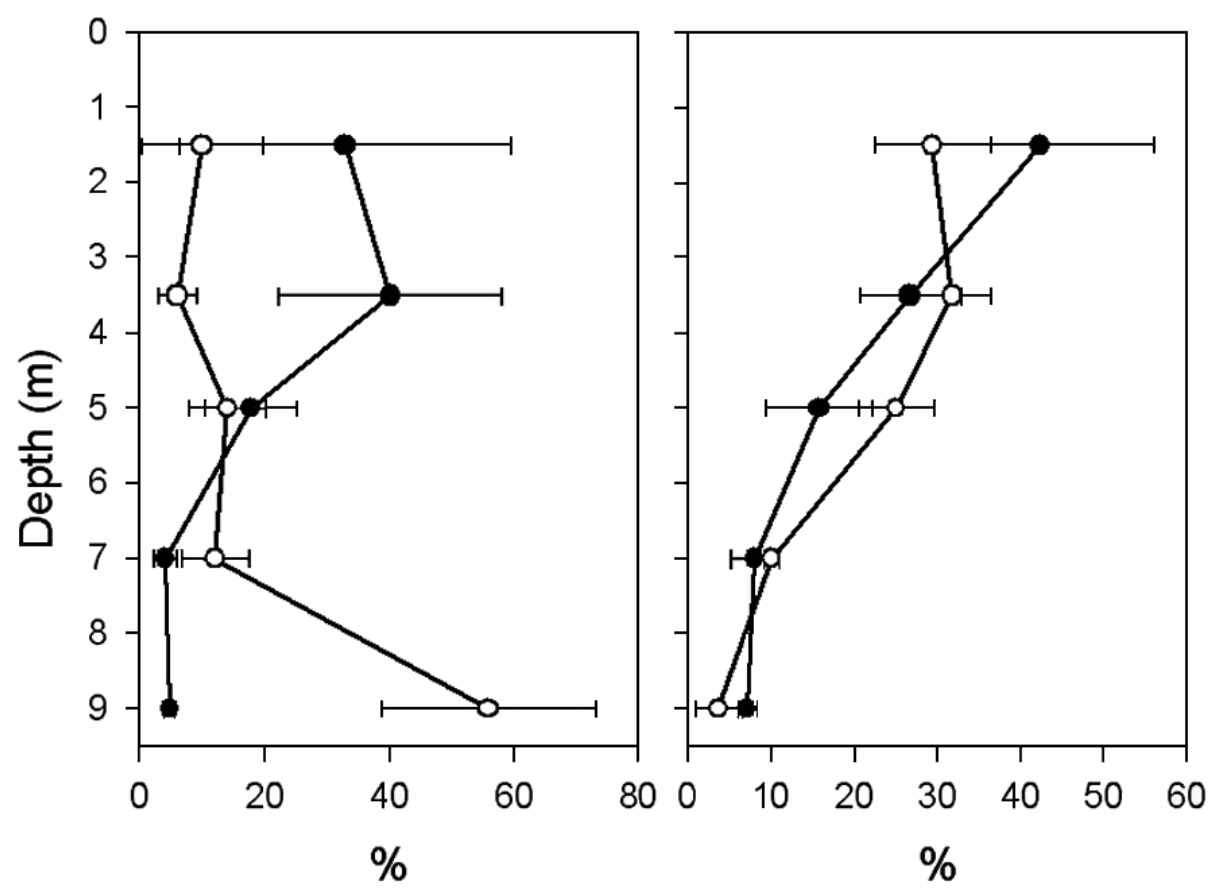

Fig. 1. Relative vertical distributions (means $\pm \mathrm{SE}, \mathrm{n}=3$ ) during day (open circles) and at night (closed circles) of $D$. pulicaria on days 15, 20 and 23 of series 1 . Left panel: tower 1 with fish; right panel: tower 2 without fish).

system (8-12 individuals/vessel) under long-day conditions (16 hours light) at a food concentration of $0.3 \mathrm{mg}$ $\mathrm{C}^{-1}$ until they were ready to deposit their fourth clutch of eggs in the brood pouch. They were then divided into two groups: one group remained under long-day conditions, the other was transferred to short-day conditions (10 hours light) before the daphniids deposited the eggs. This ensured that the eggs were laid under short photoperiod. Offsprings of both groups were then raised in the flow-through system in the respective photoperiod, hence, one group experienced the mother's photo environment, while the other was switched from long to short day. Both groups were then split further into four groups each, which differed in food and temperature conditions. The conditions were selected to mimic the environment experienced by daphniids with differing vertical migration strategies:

1) Constant food $\left(0.3 \mathrm{mg} \mathrm{C} \mathrm{^{-1 }}\right)$ and temperature (20 ${ }^{\circ} \mathrm{C}$ ) mimics daphniids residing continuously in the epilimnion.

2) Food fluctuating between $0.1 \mathrm{mg} \mathrm{C} \mathrm{l}^{-1}$ during day and $0.3 \mathrm{mg} \mathrm{C}^{-1}$ at night, but constant temperature $\left(20^{\circ} \mathrm{C}\right)$ : migration within the epilimnion.

3) Constant food $\left(0.3 \mathrm{mg} \mathrm{C}{ }^{-1}\right)$ and fluctuating temperature $\left(20{ }^{\circ} \mathrm{C}\right.$ during day, $15^{\circ} \mathrm{C}$ at night $)$ : migration without a food gradient.

4) Both food and temperature fluctuating as before: normal vertical migration.

This design resulted in 8 different treatments, each consisting of 5 replicate flow-through vessels containing
10 individuals. The daphniids were kept under these conditions for 15 days, and the proportion of females producing an ephippium was determined. In addition, we recorded survival to maturation, maturation time, size of the first clutch and female dry weight (without eggs) on day 15 .

\section{RESULTS}

\subsection{Tower experiments}

The presence of fish induced a strong diel vertical migration of D. pulicaria (Fig. 1). The majority of Daphnia in the tower with fish stayed below the thermocline during the day and ascended to the epilimnion at night, while they stayed in the epilimnion day and night in the fishless tower. Of adult females with embryos, only $5-15 \%$ were found above epilimnion, and none at a depth with light intensity more than 0.15 $\mu$ moles quanta $\mathrm{m}^{-2} \mathrm{~s}^{-1}$ or $0.1 \%$ of surface light intensity for this altitude in summer at midday.

Ephippia production in the towers was variable, but there was a consistent trend in the series depicted in figure 2. Ephippia production dropped 11-14 days after the introduction of fish. It remained low in tower 1 during the first series, when a single fish was present continuously. In both series in tower 2, ephippia production increased again 5-7 days after the fish were removed or moved close to the surface. Unfortunately, the experiment did not last long enough for another decrease in ephippia production to be seen after the towers had been restocked with fish. 

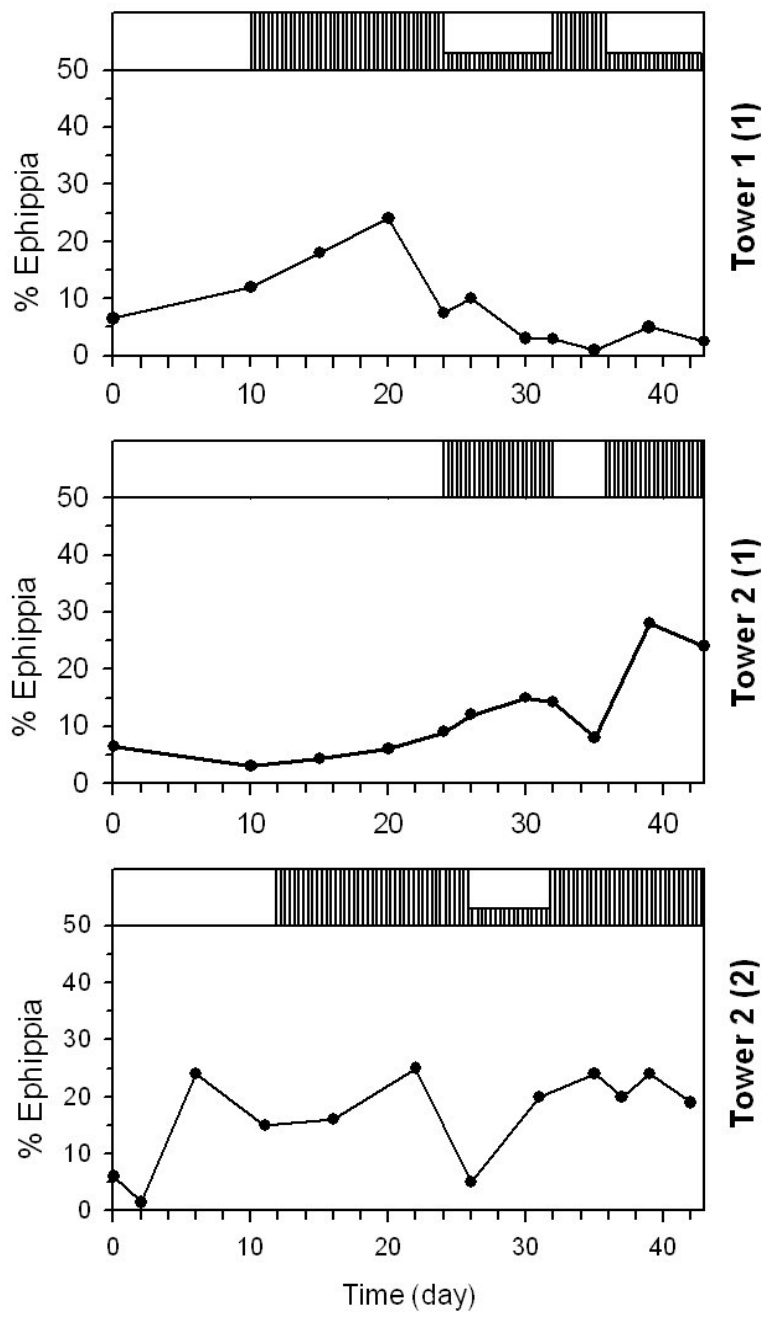

Fig. 2. Time course of ephippia production (5 ephippial females) during series 1 and 2 of the experiment. Shaded bars indicate the presence of fish. Reduced bars indicate the presence of only one fish in series 1 (tower1) or the position of the fish cage close to the surface in series 2 (tower 2).

\subsection{Reproductive status in the towers}

Proportions of ephippia in the towers and in the test jars were significantly $\left(r^{2}=0.488\right)$ correlated (Fig. 3). Application of a time lag to the tower values did not result in a higher correlation coefficient. Hence, the induction status of the females in the towers explains about $50 \%$ of the variability in the proportion of females carrying ephippia, and the direct effect of predation can only be of minor importance.

\subsection{Factors influencing ephippia production}

Survival was high in all treatments, with few exceptions $90 \%$ or more.

Although there was considerable variability in the proportion of females producing ephippia, the proportions were clearly higher when offspring lived under long day photoperiod (Tab. 1). An ANOVA with pho- toperiod, food and temperature to which offsprings were exposed as factors revealed a significant effect of photoperiod $(\mathrm{F} 1,31=15.5 ; p<0.001)$, while the effects of food and temperature were not significant.

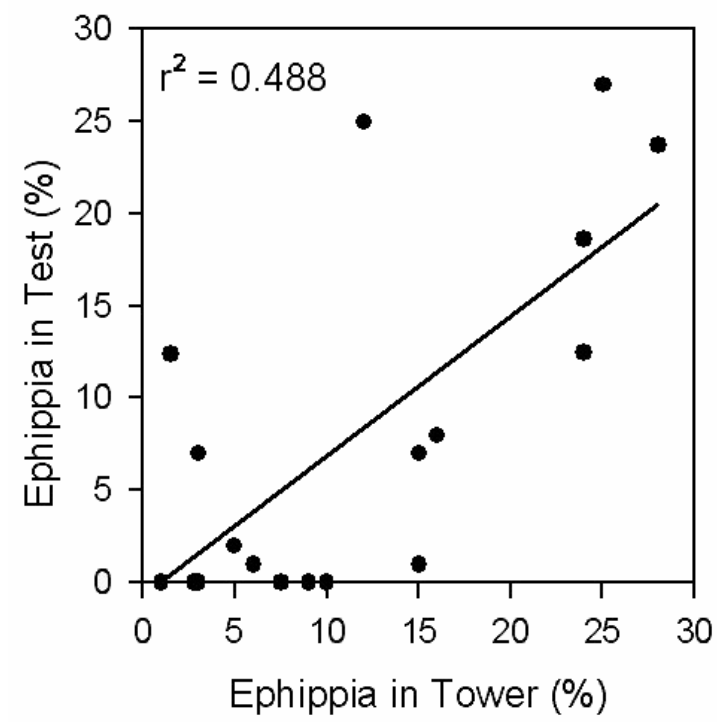

Fig. 3. Correlation between the proportion of ephippial females in the towers and the proportion of females producing ephippia in the laboratory test during the respective time periods.

Residuals for the other life-history parameters were not normally distributed, thus the factors were individually tested by Kruskall-Wallis one-way ANOVA (Tab. 2). There were few significant effects. As expected, food affected the size of the first clutch, and temperature the time to maturation. Photoperiod had a marginally significant effect on the time to maturation. None of the factors influenced the dry mass of females after 15 days.

\section{DISCUSSION}

Our first study of the influence of photoperiod changes on the maternal effect of diapause in Daphnia pulicaria resulted in an unpredictable decrease in ephippia production when individuals produced by mothers grown in long day conditions were exposed to short day conditions from the embryo stage on (Alekseev \& Lampert 2001).

As a preliminary conclusion we interpreted this result as a genetically determined adaptation of Daphnia to vertical migration induced by fish predation.

In the present study the experiments carried out in experimentally simulated temperature conditions suggested that fish mediated the vertical distribution of Daphnia pulicaria by inducing the decrease or increase of ephippia production. Laboratory experiments with flow-through-systems provided statistically significant confirmation that the most important factor affecting 
Tab. 1. Effects of offspring photoperiod, temperature and food on the proportion (\%) of females producing an ephippium (mean $\pm 1 \mathrm{SD}$ ). Maternal photoperiod was always 16:8 h (L:D). Ranges of temperature and food indicate fluctuating conditions.

\begin{tabular}{|c|c|c|c|c|}
\hline \multirow{2}{*}{$\begin{array}{l}\text { Temperature }\left({ }^{\circ} \mathrm{C}\right) \\
\text { Food }\left(\mathrm{mg} \mathrm{C} 1^{-1}\right)\end{array}$} & \multicolumn{2}{|c|}{20} & \multicolumn{2}{|c|}{$15-20$} \\
\hline & 0.3 & $0.1-0.3$ & 0.3 & $0.1-0.3$ \\
\hline \multicolumn{5}{|l|}{ Photoperiod (L:D) } \\
\hline Long day (16:8) & $9.3(9.5)$ & $13.4(7.6)$ & $15.0(14.1)$ & $26.0(5.5)$ \\
\hline Short day $(10: 14)$ & $1.8(4.0)$ & $9.2(14.5)$ & $1.8(4.0)$ & $4.8(5.5)$ \\
\hline
\end{tabular}

ephippia production in migrating Daphnia was photoperiod changes.

It is known that the embryos and the young females of Daphnia are sensitive to day length periods (Stross 1987).

Tab. 2 Effects of environmental factors on the size of the first clutch (Clutch 1), the time to maturation ( $\left.\mathrm{T}_{\text {mat }}\right)$ and the somatic dry mass of females after 15 days (Mass) of D. pulicaria (Kruskall-Wallis one-way ANOVA on ranks). Probability levels are given as numbers. NS $=$ not significant at the $5 \%$ level.

\begin{tabular}{lccc}
\hline Factor & Photoperiod & Food & Temperature \\
\hline Clutch 1 & $\mathrm{NS}$ & 0.029 & $\mathrm{NS}$ \\
$\mathrm{T}_{\text {mat }}$ & 0.043 & $\mathrm{NS}$ & $<0.001$ \\
Mass & $\mathrm{NS}$ & $\mathrm{NS}$ & $\mathrm{NS}$ \\
\hline
\end{tabular}

As signals to stop or start ephippia production in migrating or non-migrating Daphnia, I hypothesize maternal effects induced by changes in photoperiod environments: mother and embryo stay in a migrating situation (changing light conditions) or non-migrating situation (same light conditions). Although the migrating females and their embryos were at the same depth, they might be faced with different photoperiod environments. A possible explanation of this phenomenon might be a different sensitiveness of females and embryos to light intensity.

Fish presence forced Daphnia to stay at a depth with low-light conditions (in our case less than $0.02 \mu$ moles quanta $\mathrm{m}^{2} \mathrm{~s}^{-1}$, which corresponds to the night light intensity produced by moon and stars) during daytime hours to avoid being attacked by fish. Daphnia females meanwhile must constantly check light intensity changes as they go up to forage. In our experiments in the towers this was confirmed by the fact that vertical migration in Daphnia was immediately induced when small lamps were sporadically switched on and off during our experiments in the dark period (unpublished observation). Possibly this tracing for light intensity in Daphnia was realised by short time vertical jumps of females above this night-light-threshold to the depth with light intensity sufficiently high to be recognized as day light. Our observation of the vertical distribution of Daphnia in the towers showed that at any one time during the day $5-15 \%$ of adult females were found at depths with light intensity between 0.01 and $0.1 \%$ of the surface maximal light. To my mind, the critical light threshold for embryos to follow the photoperiod and enough for females to perceive the presence of light is located within these limits.

The literature points to a similar minimal light intensity necessary for diapause induction in Cladocerans. Shan (1974), who studied the influence of 25 combinations of photoperiod and light intensity on the transition to sexual reproduction in two Pleuroxus species, found by experiment that the lowest light-sensitive threshold was about 1.5 luxes or $0.06 \%$ of surface light in his conditions.

In our case, adult Daphnia migrated upwards with a light intensity of about $0.1 \%$ surface maximal light. Such a dim light occurred at between 5 and $7 \mathrm{~m}$ depth and seemed enough for Daphnia pulicaria adults to check day length.

\section{CONCLUSIONS}

1. Fish presence in the water column forced adults and young females of Daphnia pulicaria to migrate and stay during the day in lightless or dim conditions (at least below 1.5-2.0 Secchi disk depth). Ephippia production in Daphnia decreased 2-4 fold within 913 days after the beginning of vertical migration.

2. When fish were removed from the tower or located in its surface layers, there was a rapid (4-8 days) and intensive (4-6 times) increase in ephippia production.

3. The most marked effects of vertical migration on diapause induction in Daphnia pulicaria were obtained when food conditions were limited (0.2-0.3 $\left.\mathrm{mg} \mathrm{C} \mathrm{l}^{-1}\right)$. When mother and embryo are in alternating light conditions, changes in photoperiod are thought to be a signal to stop ephippia production in migrating Daphnia.

4. The light threshold for adult Daphnia and their embryos to check day length is probably within 0.1$0.02 \mu$ moles quanta $\mathrm{m}^{-2} \mathrm{~s}^{-1}$, and possibly higher for embryos.

5. Fluctuations of food conditions (from 0.3 to $0.1 \mathrm{mg}$ $\mathrm{C}^{-1}$ during daytime hours) played a relatively small role in diapause induction in "migrating" animals.

6. No significant effects of temperature fluctuation (from $20{ }^{\circ} \mathrm{C}$ to $15^{\circ} \mathrm{C}$ during daytime hours) on diapause induction in Daphnia were found.

\section{ACKNOWLEDGMENTS}

All experiments for this study were done in MaxPlanck-Institute of Limnology, Ploen, Germany in 2002. 
Prof. W. Lampert controlled food conditions in towers during the experiments and rendered an incalculable service with the manuscript preparation. This study was supported with a fellowship from Max-Planck-Society and a research grant from Russian Foundation for Basic Researches (N 04-04-49121 RFBR).

\section{REFERENCES}

Alekseev, V. \& W. Lampert. 2001a. Maternal control of resting-egg production in Daphnia. Nature, 414: 899-901.

Alekseev, V. \& W. Lampert. 2001b. Maternal experience in photoperiod can affect diapause response in Daphnia offspring. Abst. IVth European Workshop of Invertebrate Ecophysiology. St.Petersburg, Russia, 9-15 September 2001: 43-44

Caceres, C. E. 1998. Interspecific variation in the abundance, production, and emergence of Daphnia diapausing eggs. Ecology, 79: 1699-1710.

Hairston, N.G.J. 1996. Zooplankton egg banks as biotic reservoirs in changing environments. Limnol. Oceanogr., 41: 1087-1092.

Hutchinson, G. E. 1967. A treatise on limnology. II. Introduction to lake biology and the limnoplankton. Wiley, New York: $1115 \mathrm{pp}$.

Kleiven, O.T., P. Larsson \& A. Hobaek. 1992. Sexual reproduction in Daphnia magna requires three stimuli. Oikos, 65: 197-206.

Lampert, W., 1987. Feeding and nutrition in Daphnia. Mem. Ist. ital. Idrobiol., 45: 143-192.

Lampert, W. 1993. Ultimate causes of dial vertical migration of zooplankton: new evidence for the predator avoidance hypothesis. Arch. Hydrobiol. Beih. Ergebn. Limnol., 39: 79-88.
Lampert, W. \& C. J. Loose. 1992. Plankton towers: Bridging the gap between laboratory and field experiments. Arch. Hydrobiol., 126: 53-66.

Mellors, W. K., 1975. Selective predation of ephippial Daphnia as and the resistance of ephippial eggs to digestion. Ecology, 56: 974-980.

Pijanowska, J. \& G. Stolpe. 1996. Summer diapause in Daphnia as a reaction to the presence of fish. J. Plankton Res., 18: 1407-1412.

Slusarczyk, M. 1995. Predator-induced diapause in Daphnia. Ecology, 76: 1008-1013.

Slusarczyk, M. 2004. Environmental plasticity of fish avoidance diapause response in Daphnia magna. J. Limnol., 63 (Suppl. 1): 70-74.

Sommer, U., Z.M. Gliwicz, W. Lampert \& A. Duncan. 1986. The PEG model of seasonal succession of planktonic events in fresh waters. Arch. Hydrobiol., 106: 433-471.

Spaak, P. 1995. Sexual reproduction in Daphnia: interspecific differences in a hybrid species complex. Oecologia, 104: 501-507.

Spaak, P. \& M. Boersma. 2001. The influence of fish kairomones on the induction and vertical distribution of sexual individuals of the Daphnia galeata species complex. Hydrobiologia, 442: 185-193.

Stross, R.G. 1987. Photoperiodism and phased growth in Daphnia populations: coactions in respective. Mem. Ist. ital. Idrobiol., 45: 413-437.

Stross, R.G. \& R. G. Hill. 1965. Diapause induction in Daphnia requires two stimuli. Science, 150: 1462-1464.

Taylor, F. 1980. Optimal switching to diapause in relation to winter. J. theor. Biol., 18: 125-133.

Tollrian, R. \& C. D. Harvell (Eds). 1999. The ecology and evolution of inducible defences. Princeton University Press, Princeton.

Zehnder, A.A. \& P.R. Gorham. 1960. Factors influencing the growth of Microcystis aeruginosa Kütz. emend. Elenk. Can. J. Microbiol. 6: 645-660. 